\title{
Application of Fractional Calculus to Modeling Transient Combustion of Solid Propellants
}

\author{
Vladimir Kulish ${ }^{1, \text { a) }}$, Vladimír Horák ${ }^{2, b)}$, Linh Do Duc ${ }^{2, \text { c) }}$ and Tomáš Lukáč ${ }^{2, \text { d) }}$ \\ ${ }^{1}$ School of Mechanical \& Aerospace Engineering, Nanyang Technological University, Singapore 639798 \\ ${ }^{2}$ Faculty of Military Technology, University of Defense, Czech Republic. \\ ${ }^{a)}$ Corresponding author: mvvkulish@ntu.edu.sg \\ b) vladimir.horak@unob.cz \\ c)duclinh.do@gmail.com \\ d) tomas.1ukac@unob.cz
}

\begin{abstract}
It was Zel'dovich, who first considered the transient combustion problem of solid propellants. Some more detailed models of that process have been developed afterwards. However, until today, numerical methods remain the prevailing tool for modeling unsteady combustion processes. In this work, it has been demonstrated that at least one of the problems of the unsteady combustion theory, which previously investigated numerically, can be treated analytically by means of fractional calculus. The solution for the unsteady speed of combustion thus derived is then compared with the solution obtained by numerical means in previous studies. The comparison shows a good agreement between those results, especially for small values of time.
\end{abstract}

\section{INTRODUCTION}

The transient combustion problem of solid propellants was first considered by Zel'dovich [1], after which some more detailed models of that process have been developed [2]. Until nowadays, however, numerical methods remain the prevailing tool for modeling unsteady combustion processes.

In this work, it has been demonstrated that at least one of the problems of the unsteady combustion theory, which previously investigated numerically, can be treated analytically by means of fractional calculus.

The solution for the unsteady speed of combustion thus derived is then compared with the solution obtained by numerical means in previous studies. The comparison shows a good agreement between those results, especially for small values of time.

\section{THE METHOD}

Consider one of the problems in the theory of transient combustion, which has been previously investigated by numerical methods in [2]:

$$
\begin{gathered}
{\left[\frac{\partial}{\partial \tau}-\frac{\partial^{2}}{\partial \xi^{2}}-\omega(\tau) \frac{\partial}{\partial \xi}\right] \Theta=0, \quad 0<\xi<\infty, \quad 0<\tau<\infty} \\
\left.\frac{\partial}{\partial \xi}\right|_{\xi=0}=\left[\left(\frac{\omega \eta}{\pi^{2}}-1\right)^{2}-1\right] \frac{\pi^{v}}{2 \eta-\eta^{2}} \\
\left.\Theta\right|_{\xi=0}=1 ;\left.\quad \Theta\right|_{\xi=\infty}=0 ;\left.\quad \Theta\right|_{\tau=0}=e^{-\xi} \\
\left.\pi\right|_{\tau=0}=1 ;\left.\quad \omega\right|_{\tau=0}=1
\end{gathered}
$$


The nomenclature is borrowed from [2] for the sake of consistency. The dimensionless quantities are given by the following expressions, where $u$ denotes the speed of combustion, $a$ is the thermal diffusivity of the condensed phase, $T$ is the temperature in an arbitrary cross-section, $T_{1}$ and $T_{0}$ stay for the temperature values on the surface of combustion and far away from the reaction zone, respectively, while $\alpha$ and $v$ are empirical constants in the law of steady combustion.

It is assumed that combustion of the condensed matter initially takes place at pressure $P_{0}$ with a constant linear speed $u_{0}$. At the initial time moment $\tau=0$, a pressure jump to the value $P_{1}$ takes place. The unsteady value of the combustion speed $\omega(\tau)$ is to be found for all $\tau>0$.

Thus, the quantities $\Theta(\xi, \tau)$ and $\omega(\tau)$ in Eqn. (1) are variable, while the quantities $\eta$ and $\pi^{v}$ are given.

Upon introducing a new variable $\theta=\Theta-e^{-\xi}$, the initial conditions become homogeneous (zero) and Eqn. (1) can be written as

$$
\begin{gathered}
{\left[\frac{\partial}{\partial \tau}-\frac{\partial^{2}}{\partial \xi^{2}}-\omega(\tau) \frac{\partial}{\partial \xi}\right] \theta=e^{-\xi}[1-\omega(\tau)]} \\
\left.\frac{\partial \theta}{\partial \xi}\right|_{\xi=0}-1=\left[\left(\frac{\omega \eta}{\pi^{v}}-1\right)^{2}-1\right] \frac{\pi^{v}}{2 \eta-\eta^{2}} \\
\left.\theta\right|_{\xi=0}=0 ;\left.\quad \theta\right|_{\xi=\infty}=0 ;\left.\quad \theta\right|_{\tau=0}=0 \\
\left.\pi\right|_{\tau=0}=1 ;\left.\quad \omega\right|_{\tau=0}=1
\end{gathered}
$$

The value of the derivative at the boundary $\xi=0$ for Eqn. (2) can be obtained as follows.

Consider the problem:

$$
\begin{gathered}
{\left[\frac{\partial}{\partial t}-\alpha(x, t) \frac{\partial^{2}}{\partial x^{2}}-\beta(x, t) \frac{\partial}{\partial x}+v(x, t)\right] T=Q(x, t), 0<x<\infty, \quad 0<t<\infty} \\
\left.T\right|_{x=0}=T_{S}(t) ;\left.\quad T\right|_{x=\infty}=0 ;\left.\quad T\right|_{t=0}=0
\end{gathered}
$$

Equation (3) can be rewritten as

$$
\left[\sum_{m=0}^{\infty} b_{m}(x, t) D^{\frac{1-m}{2}}-B(x, t) \frac{\partial}{\partial x}\right]\left[\sum_{n=0}^{\infty} a_{n}(x, t) D^{\frac{1-n}{2}}+A(x, t) \frac{\partial}{\partial x}\right] T=Q(x, t)
$$

Or, in the operator form, as

$$
M L T=Q
$$

where the operators $M$ and $L$ become clear from comparing Eqns. (4) and (5).

Now, instead of Eqn. (5), consider

$$
L T=Q^{*}
$$

where $Q^{*}$ is an auxiliary function such as

$$
M Q^{*}=Q
$$

Solutions of Eqn. (6) are also the solutions of Eqn. (5). This can be established by multiplying the left side of Eqn. (7) by $M$. Note also that the solutions of Eqn. (6) satisfy the conditions $T(x=\infty)=0$ and $T(t=0)=0$. Hence, upon writing Eqn. (6) at $x=0$, one obtains an expression that defines the gradient on the boundary, namely: 


$$
-A(0, t) q_{s}(t)=\sum_{n=0}^{\infty} a_{n}(0, t) D^{\frac{1-n}{2}} T_{S}-Q^{*}(x, t)
$$

As regards the auxiliary function $Q^{*}$ from Eqn. (7), it is obvious that $Q^{*}=M^{-1} Q$, where $M^{-1}$ can be found in the form of a series

$$
M^{-1}=\sum_{n=0}^{\infty} E_{n}\left(x, t, \frac{\partial^{k}}{\partial x^{k}}\right) D^{-\frac{1-n}{2}}, \quad k \leq n
$$

where $E_{n}$ are still unknown operators dependent on $x, t$, and the derivatives with respect to $x$. After substituting Eqn. (9) into

$$
M M^{-1} f=f
$$

one can obtain the set of equations, from which $E_{n}$ can be found as

$$
\begin{aligned}
& E_{0}=1 \\
& E_{1}=\sqrt{\alpha} \frac{\partial}{\partial x}-b_{1} \\
& E_{2}=\sqrt{\alpha} \frac{\partial}{\partial x} E_{1}-b_{1} E_{1}-b
\end{aligned}
$$

The operators $M$ and $L$ in Eqn. (5) are given now by

$$
\begin{aligned}
& M=\left[D^{\frac{1}{2}}-\frac{\omega}{2}+\frac{\omega^{2}}{8} D^{-\frac{1}{2}}-\frac{\dot{\omega}}{8} D^{-1}-\left(\frac{\omega^{4}}{128}+\frac{\omega \dot{\omega}}{16}\right) D^{-1}+\ldots-\frac{\partial}{\partial \xi}\right] \\
& L=\left[D^{\frac{1}{2}}-\frac{\omega}{2}+\frac{\omega^{2}}{8} D^{-\frac{1}{2}}-\frac{\dot{\omega}}{8} D^{-1}-\left(\frac{\omega^{4}}{128}+\frac{\omega \dot{\omega}}{16}\right) D^{-1}+\ldots+\frac{\partial}{\partial \xi}\right]
\end{aligned}
$$

and $Q=e^{-\xi}(1-\omega)$, whereas the dots above symbols denote derivatives with respect to time.

In the case under consideration, the operator $M^{1}$ can be found from

$$
\left(D^{\frac{1}{2}}-\frac{\omega}{2}+\frac{\omega^{2}}{8} D^{-\frac{1}{2}}-\frac{\dot{\omega}}{8} D^{-1}-\ldots-\frac{\partial}{\partial \xi}\right) M^{-1}=1
$$

One obtains

$$
\begin{aligned}
M^{-1}= & D^{-\frac{1}{2}}+\left(\frac{\omega}{2}+\frac{\partial}{\partial \xi}\right) D^{-1}+\left(\frac{\omega^{2}}{8}+\omega \frac{\partial}{\partial \xi}+\frac{\partial^{2}}{\partial \xi^{2}}\right) D^{-\frac{3}{2}}+ \\
& +\left(-\frac{\dot{\omega}}{8}+\frac{\omega^{2}}{2} \frac{\partial}{\partial \xi}+\frac{3}{2} \omega \frac{\partial^{2}}{\partial \xi^{2}}+\frac{\partial^{3}}{\partial \xi^{3}}\right) D^{-2}+\ldots
\end{aligned}
$$

Then, the relation between the values of the temperature gradient on the boundary and the unsteady combustion speed becomes 


$$
\left.\frac{\partial \Theta}{\partial \xi}\right|_{\xi=0}=\left[D^{-\frac{1}{2}}+\left(1-\frac{\omega}{2}\right) D^{-1}+\left(\frac{\omega^{2}}{8}-\omega-1\right) D^{-\frac{3}{2}}+\left(-\frac{\dot{\omega}}{8}-\frac{\omega^{2}}{2}+\frac{3}{2} \omega-1\right) D^{-2}+\ldots\right](1-\omega)
$$

Upon eliminating $(\partial \vartheta / \partial \xi)_{\xi=0}$ between Eqns. (2) and (15), one obtains the equation for the unsteady speed of combustion $\omega(\tau)$ :

$$
\begin{aligned}
{\left[\left(\frac{\omega \eta}{\pi^{v}}-1\right)^{2}-1\right] \frac{\pi^{v}}{2 \eta-\eta^{2}}+1=} & {\left[D^{-\frac{1}{2}}+\left(1-\frac{\omega}{2}\right) D^{-1}+\left(\frac{\omega^{2}}{8}-\omega-1\right) D^{-\frac{3}{2}}\right.} \\
& \left.-\left(\frac{\dot{\omega}}{8}+\frac{\omega^{2}}{2}-\frac{3}{2} \omega+1\right) D^{-2}+\ldots\right](1-\omega)
\end{aligned}
$$

Because the latter expression contains fractional derivative operators of order $1 / 2$, which transform a power function into a power function, it is obvious that the solution is to be found in the form of a power series with respect to $\tau^{1 / 2:}$

$$
\omega=\sum_{n=0}^{\infty} c_{n} \tau^{\frac{n}{2}}, \quad c_{n}=\text { const }
$$

Upon substituting Eqn. (17) into (16) and equating the coefficients at the same powers of $\tau$, one obtains the expressions for the coefficients $c_{n}$ :

$$
\begin{aligned}
c_{0}= & \left\{1+\left[1-\left(2 \eta-\eta^{2}\right) \pi_{1}^{-v}\right]^{\frac{1}{2}}\right\}\left(\frac{\pi_{1}^{v}}{\eta}\right) \\
c_{1}= & \left(1-c_{0}\right)(2-\eta)\left(\eta \pi_{1}^{-v} c_{0}-1\right)^{-1} \Gamma^{-1}\left(\frac{1}{2}\right) \\
c_{2}= & {\left[\left(1-c_{0}\right)\left(c_{0}-2\right)-\Gamma\left(\frac{1}{2}\right) c_{1}-\eta \pi_{1}^{-v}(2-\eta)^{-1} c_{1}^{2}\right]\left(\frac{1}{4}\right)(2-\eta)\left(\eta \pi_{1}^{-v} c_{0}-1\right)^{-1} } \\
c_{3}= & \left\{-\Gamma^{-1}\left(\frac{5}{2}\right) c_{2}-2 \eta(2-\eta)^{-1} c_{1} c_{2} \pi_{1}^{-v}+\left(11-7 c_{0}\right)\left(\frac{c_{1}}{12}\right)++\Gamma^{-1}\left(\frac{5}{2}\right)\left(1-c_{0}\right) \mathrm{x}\right. \\
& \left.\mathrm{x}\left[1-c_{0}+\left(\frac{c_{0}^{2}}{8}\right)\right]\right\}\left[1-\left(\frac{\eta}{2}\right)\right]\left(\eta \pi_{1}^{-v}-1\right)^{-1}
\end{aligned}
$$

The positive sign of the square root in the first expression of (18) is to be chosen, because an absurd conclusion follows otherwise - the combustion speed with an increase of pressure becomes less than the initial one.

\section{MODEL VALIDATION}

For the numerical example considered in [3], $\eta=1.15 ; v=2 / 3 ; \pi_{1}=2$, after using Eqns. (17) and (18), one obtains

$$
\frac{\omega}{\omega_{1}}=1.41-0.60 \tau^{\frac{1}{2}}-0.02 \tau+0.35 \tau^{\frac{3}{2}}-\ldots
$$

The steady speed of combustion and pressure at $\tau \rightarrow \infty$ are related as $\omega_{1}=\pi_{1}^{\nu}$. 
From comparison with the result obtained in [3], one can draw the following conclusion: the less the value of $\tau$ is, the less the difference between the calculated values of $\omega / \omega_{1}$ is (it becomes zero at $\tau=0$ ). At the same time, Eqn. (19) delivers the values that are smaller than the values obtained in the course of numerical simulations. The error at $\tau=0.5$ does not exceed five per cent. For large values of $\pi_{1}$, the result obtained from Eqn. (19) becomes less certain.

\section{CONCLUSIONS}

The methods of fractional calculus, applied here to render an analytical solution to the transient combustion problem of solid propellants, seem to be a powerful tool for solving many a problem in the area of heat and mass transfer. In particular, in this work the solution for the unsteady speed of combustion has been derived and is then compared with the solution obtained by numerical means in previous studies. The comparison shows a good agreement between those results, especially for small values of time.

\section{ACKNOWLEDGMENTS}

This work has been supported by the grant from Ministry of Education, Singapore. Grant number RG119/15, the institutional funding DZRO K201 "VÝZBROJ" and by the specific research project of Faculty of the Military Technology SV16-216.

\section{REFERENCE}

1. Ya. B. Zel'dovich, O. I. Leypunskiy, and V. B. Librovich, Theory of unsteady combustion of solid propellants, (Nauka, 1975), $132 \mathrm{p}$.

2. B. V. Novozhilov, Unsteady combustion of solid propellants, (Nauka, 1973), $176 \mathrm{p}$.

3. B. V. Novozhilov, Unsteady combustion of solid propellants, (Applied Mechanics and Technical Physics, 1962, Vol. 5), pp. 83-88. 